\title{
Dynamic Eye Misalignment Retroversion System (DEMRS)
}

\author{
Constantinos M. Glynos, Dr. Olusola O. Aina and Dr. Hammadi Nait-Charif
}

\begin{abstract}
Strabismus is a medical term used to define the eye misalignment conditions that prevent both eyes from focusing on the same target simultaneously. This is a disability that prohibits the perception of depth. The purpose of going through treatment is to realign the "bad" (strabismic) eye, so that it fixates on the same target as the "good" (correct) eye. This paper presents a novel system that can dynamically adjust the light rays coming into each eye in order to stimulate both foveae, with the correct image, simultaneously. This system is to be used in diagnosis and treatment for children with strabismus under the age of eight and for patients of any age suffering from Convergence Insufficiency (CI) or Diplopia.
\end{abstract}

Index Terms-Strabismus, Squint, Diplopia, Convergence Insufficiency, Eye Misalignment, Light Redirection, Dynamic System, Diagnosis, Treatment.

\section{INTRODUCTION}

Vision is a luxury we take for granted every day without considering the consequences, should we lose it.

When a light ray hits the cornea, it travels through the aqueous humor, then gets refracted by the lens of the eye and travels through a gel like substance called the vitreous humor until it intersects with the retina [4]. The retina converts the gathered light into electrical impulses, transmitting them to the brain through the optic nerve [4][5]. The fovea is a small region at the center of the retina, and is responsible for one's crystal clear vision [5].

Barlow et al (1967) and Hubel (1995) explain that the stimuli in each eye is a $2 \mathrm{D}$ projection of the real world, due to the monocular visual field of each eye [9][5]. The horizontal and vertical distance between the eyes, justify that the brain receives two slightly different angled images which needs to fuse in one [1][5]. The difference between these two images is known as the binocular disparity [1][5].

The greatest traits of this process, known as binocular vision, is the acquisition of depth, measurement of distance and stereopsis[1][9]. The basis of binocular vision is known as Retinal Correspondence and is defined by the stimulation of both retinas from the same point in the horopter space [6]. In order for someone to accurately perceive depth, stereopsis (stereoscopic vision) must be present and the target object in space needs to successfully stimulate both foveae [7]. Additionally, the binocular disparity should cause a horizontal displacement of no more than $2^{\circ}$ and a vertical displacement of nearly zero [1]. Furthermore, one must be able to process familiarity cues such as relative sizes, orientation of objects, shadows, motion parallax, occlusion and perspective [5][9].

Unfortunately not everyone can perceive depth in the same manner. When the eyes are not properly aligned and do not point at the same target simultaneously, the retinas get stimulated by $2 \mathrm{D}$ projections that are widely different preventing the brain from fusing them properly [1][5]. The root behind this occurrence is still medically unknown while the reasons vary from patient to patient [1]. Strabismus is the medical term used to distinguish any eye misalignment condition defined by the inability of the eyes to focus on the same target simultaneously [3][6].

Three well known categories of strabismus are Squint, Diplopia and Convergence Insufficiency (CI) [1][3][6]. In the case of a Squint, the brain selectively projects a scotoma onto the fovea of the "bad" eye to avoid any permanent damage, such as Constant Diplopia (double vision) [1]. In both CI and Diplopia, the brain is able to fuse the two images but due to the eye misalignment factor the patient sees double or has blurred vision [6][10]. Barlow et al (1967), Damanakis (2013) and Hammond (2014) assert that in the case of monocular vision, one uses only familiarity cues to calculate the depth and estimate distances which "require rather complex image-processing" (Barlow et al, 1967) [9][6][3].

Cooper (2012) claims that, 5\% of the population in the United States suffer from CI [18], while Damanakis (2013) advances that about $7 \%$ of the people worldwide suffer from a Squint [1].

According to Damanakis (2013) and Hammond (2014), the sooner strabismus gets diagnosed, the higher the chances of treatment success [1][3]. In all cases, the first test is to clarify the visual acuity for far objects $(6 \mathrm{~m})$ and near targets $(0.33 \mathrm{~m}-0.6 \mathrm{~m})$ [2][8] at different directions [2]. This test will also determine the type of strabismus, ie. esotropia, exotropia, hypertropia, hypotropia, incyclotropia or excyclotropia [3][6].

The haploscope is a diagnostic device that helps measuring strabismus angles through the use of two images, e.g. a lion on the left side and a cage on the right side, and lets the patient "place" the lion inside the cage by adjusting the angle and position of each side manually [6]. Treating strabismus depends upon the patient's age and the deficiency's origin. In the case of CI and diplopia, were binocular vision is present, treatment can take place at any age without any fear of diplopia [1]. Patients with squints however, can undergo therapy up until the age of 7 or 8 years old [1][3]. 
The most common treatments are pencil push ups, eye patching, prism glasses, botox, computerized vision therapy, and surgery [1][8][11]. Pencil push ups try to improve the signaling between the brain and the "bad" eye [1]. This exercise is recommended to CI patients however it is not a very effective approach [10][12]. Eye patching is not a method for treating strabismus, however it is the oldest yet most reliable method for treating amblyopia, as it forces the brain to use the "bad" eye as the main source of vision [6]. Base-in prism glasses are used mostly in CI and diplopia and are considered to be "a passive, compensatory treatment"[10][12]. In 1981, Alan Scott published his results after using botox on strabismus patients to weaken the muscles of the "bad" eye and re-align it [15]. In practice however, botox treatments do not last for long, cannot be injected into children and is only used for aesthetic reasons [1]. Home and office based Vision Therapy (VT) is highly recommended for CI patients as it offers a high percentage of treatment success [8][13]. Recently, computer games have entered the area of VT promoting the motivation of the patient to complete the exercises [14]. According to specialists in the field of strabismus, surgery is the most recommended option [1][3]. However, even with this accomplishment, binocular vision cannot be restored because as soon as the patient fixates on either side other than straight ahead, the "bad" eye deviates causing suppression [1][3]. The percentage of binocular fusion and stereoscopic success from surgery is extremely low [1]. This is the reason why surgery is mostly performed for aesthetic reasons [1][3].

The purpose of this research is to explore new ways for diagnosing and treating strabismus. According to specialists, currently, there is no system that can continuously redirect the foveal light rays in order to compensate strabismus angles [1][2][3].

\section{Dynamic Eye Misalignment Retroversion System (DEMRS)}

\subsection{Description and Design of the System}

Unlike haploscopes, which adjust the images projected onto the two foveae, or surgery, where doctors adjust the extraocular muscles, the Dynamic Eye Misalignment Retroversion System (DEMRS) is a head mounted device which redirects light. It supports the simultaneous stimulation of the two foveae by adjusting the direction of light rays entering the eyes (Fig. 1).

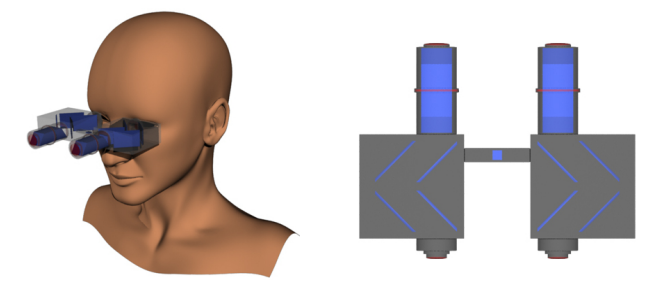

Figure 1: DEMRS Overview
A potential blueprint of this device (Fig. 2) illustrates its composition out of four cold mirrors $\left(\mathrm{M}_{\mathrm{L} / \mathrm{R}_{1}}, \mathrm{M}_{\mathrm{L} / \mathrm{R}_{2}}\right.$, $\left.\mathrm{M}_{\mathrm{L} / \mathrm{R}_{3}}, \mathrm{M}_{\mathrm{L} / \mathrm{R}_{4}}\right)$ and two dove or delta prisms $\left(\mathrm{P}_{\mathrm{L} / \mathrm{R}}\right)$ on each side compensating for re-alignment. The prisms are added only for patients that suffer from torsional strabismus. The two infra-red sensitive cameras $\left(\mathrm{C}_{\mathrm{L} / \mathrm{R}}\right)$ are placed behind the $\mathrm{M}_{\mathrm{L}_{1}}$ and the $\mathrm{M}_{\mathrm{R}_{1}}$ tracking the positions of the eyes. In addition, the accelerometer (Accel) measures the tilting angle of the structure providing information for the patient's head movement.

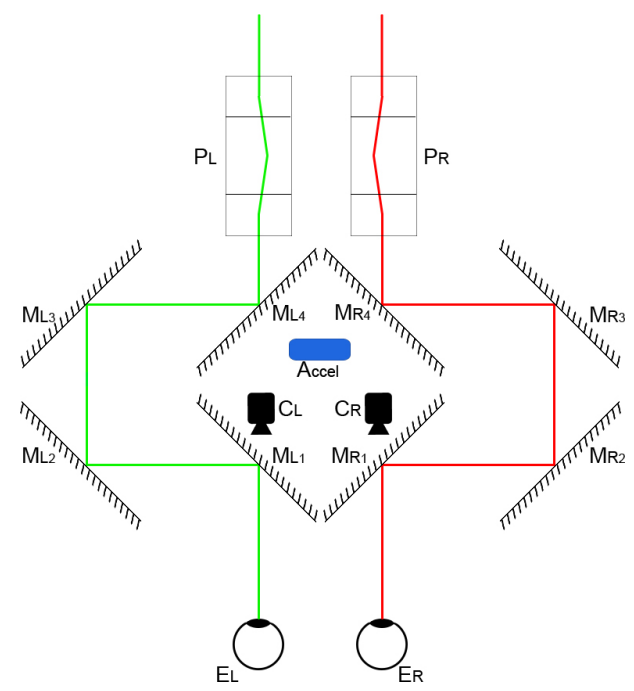

Figure 2: System Overview

The Dove prisms or a Delta prisms (compact version of a dove) have the ability to invert and rotate the transmitted image at twice the rate of prism rotation [18]. The combination of such prisms, one in front of the other, allows the image to be rotated without causing any inversion. The patient's eyes will be tracked by two infra-red sensitive cameras placed behind the first set of mirrors. In order to accomplish that, the mirrors need to be of a certain type which allow infra-red light to be transmitted. Such mirrors are known as cold mirrors that can reflect $90 \%$ of the visible light and transmit $80 \%$ of infra-red light [17]. Head tilts cause torsional rotation of the eyes (incyclotropia and excyclotropia) which the device can track using an accelerometer mounted at it's center.

According to Damanakis (2004) and Hammond (2014), strabismus angles can measure up to $45^{\circ}$ horizontally, $20^{\circ}$ vertically and about $10^{\circ}-15^{\circ}$ torsionally [6][3]. To facilitate a wider field of view for the eyes, as well as preventing the mirrors from colliding when rotating inwards, each mirror's pivot of rotation was translated on to it's side instead of the center. Figure $3 \mathrm{a}$ and figure $3 \mathrm{~b}$ illustrate a case where the mirror needs to rotate by $15^{\circ}$.

In addition, the system retroverses the foveal light ray (Vision Vector, VV) at the center of $\mathrm{M}_{\mathrm{L} / \mathrm{R}_{2}}$ and $\mathrm{M}_{\mathrm{L} / \mathrm{R}_{3}}$.

The DEMRS's applications revolve around diagnosis and treatment. For this reason, it is essential that the fixation target is either known (set by the ophthalmologist) or deduced from the eye angles [1]. Damanakis (2013) explains 


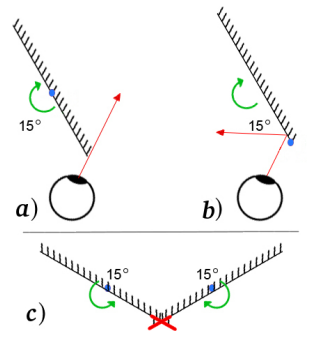

Figure 3: Rotation Pivot

the techniques currently used for estimating the fixation distance through one's accommodative convergence [2].

Accommodation (A) is the amount of energy applied by the eye lens to fixate upon a near object $(<6 m)$ (Fixation Distance $=$ FD) $($ Eq. 1) [6].

$$
A_{(\Delta)}=\frac{1}{F D_{(m)}}
$$

For far objects $(\geqslant 6 m)$, the eye lens does not need to accommodate [6]. In order to accomplish bifoveal stimulation on a near target object, both eyes need to rotate so that their foveal vectors intersect at that point. This is known as convergence $(\mathrm{C})$ which is relative to the distance of the object and each individual's disparity distance $(\delta)$ (Eq. 2) [6].

$$
C_{(\Delta)}=\frac{1}{F D_{(m)}} \times \delta_{(\mathrm{cm})}
$$

Accommodative convergence (AC) is the relative amount of accommodation applied by the "bad" eye as the "good" eye fixates upon an object [6]. According to Damanakis, the target's distance can be found using the accommodative convergence factor along with the ratio $(A C / A)[6]$.

The target's location in a 3D space, would still remain unknown as the distance variable could be related to any number of directions. Knowing the disparity (Fig. 4a), the "good" eye's direction vector $\left(V V_{R}\right)$ and the fixation distance (Fig. 4b, 4c), it is possible to find the correct direction vector $\left(V V_{L}\right)$ by subtracting the end point of the "good" eye's side from the position of the "bad" eye (Fig. 4d).

Assuming that the fixation point is either known, or computed, the system would adjust itself so that both foveal light vectors could converge on the same target simultaneously. Two auxiliary vision vectors, one for each eye, are created in order to analyze the patient's original vision. In case of misalignment, the system determines their correct direction. If the bifoveal rays fail to intersect on the target point, then the strabismus is identified and the corrected vision vector are computed. Furthermore, the system adjusts the angles of the mirrors and prisms so that the final reflected rays from the device, match the auxiliary vectors.

\subsection{Retroversion Algorithm}

The main purpose of going through one or more strabismus treatments is to realign the "bad" eye, so that it fixates on the same target as the other eye [1]. In simple terms, the
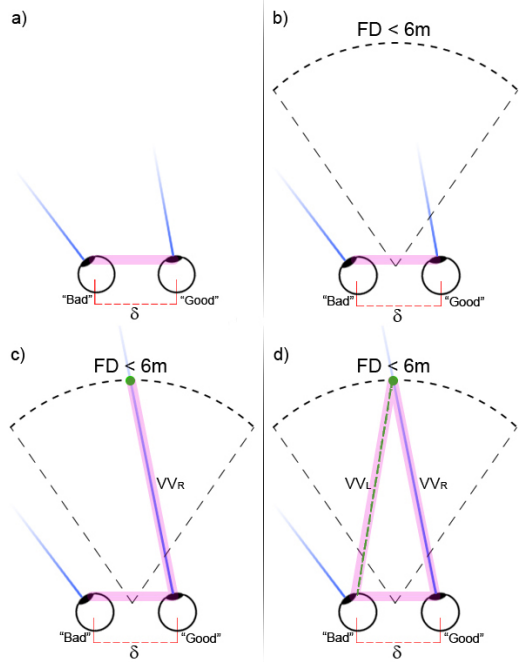

Figure 4: Target Deduction

task is to make the "bad" eye fixate upon point $B$ rather than point $G$ (Fig. 5).

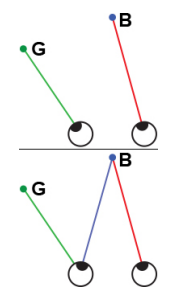

Figure 5: From point $\mathrm{G}$ to $\mathrm{B}$

In the heart of the DEMRS, lies a deterministic geometric algorithm which retroverses the direction of the foveal light ray via a set of mirrors to accomplish the above task.

According to the law of reflection (Fig. 6a), the reflected angle $(\theta r)$ is equal to the incident angle $(\theta i)$ defined by the angle between the incident ray $\left(\overrightarrow{I_{A}}\right)$ and the normal vector of the planar surface $(\vec{N})$. Furthermore, when rotating a mirror by an angle $\phi$ around $P$, the reflected ray will rotate by twice of that angle $(2 \phi)$.

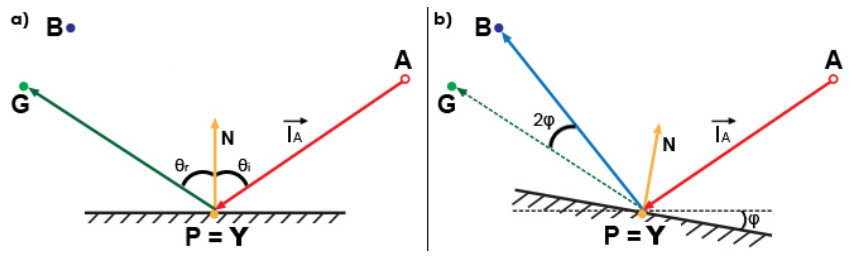

Figure 6: Rotating a mirror from the point of intersection

Both $G$ and $B$ are known $3 \mathrm{~d}$ points and $Y$ is the point of intersection of the incident ray $\overrightarrow{I_{A}}$ with the mirror surface (also equal to it's rotation pivot $P$ ) (Fig. 6b).

The mirrors in the design would need to rotate about the side (Fig. 7a). Therefore, the previous method will not produce the correct results (Fig. 7b) as the point of intersection is no longer equal to the pivot of rotation $(Y \neq P)$. 


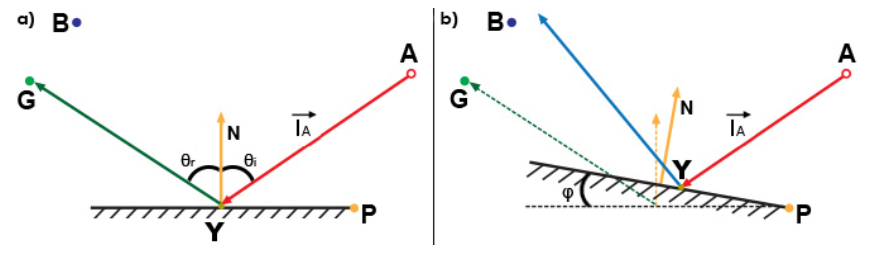

Figure 7: Rotating a mirror from a pivot (problem)

We find the rotation angle $\phi$,in a 2-dimensional coordinate system, of the mirror around a pivot $P$, so that when the incident vision vector $\overrightarrow{I_{A}}$ hits the surface at point $Q$, it creates a reflection vector $\vec{R}_{Q B}$ that intersects point $B$ (Fig. 8).

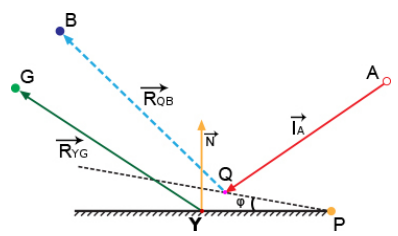

Figure 8: 2D Retroversion problem

Since this is a physically based system, it entails that only one angle $\phi$ may exist that can produce the desired result. Rotating the mirror around a pivot other than from where the ray intersects the surface, creates a circle with center $P$ and an unknown radius $r_{p}$ (Fig. 9).

When $\phi=0^{\circ}$, the reflection vector $R_{Y G}$ is a tangent $\overrightarrow{T_{G_{1}}}$ to the circle. Computing the point of tangency $G^{\prime}$ and calculating the distance from $P$, gives the radius $r_{p}$ of the circle (Fig. 9).

$$
\begin{gathered}
\overrightarrow{T_{G_{1}}}=-\overrightarrow{R_{Y G}} \\
u=\frac{\overrightarrow{G P} \odot T_{G_{1}}}{\overrightarrow{T_{G_{1}} \odot T_{G_{1}}}} \\
G^{\prime}=G+u \times \overrightarrow{T_{G_{1}}} \\
r_{p}=\mathrm{d}\left(P, G^{\prime}\right)
\end{gathered}
$$

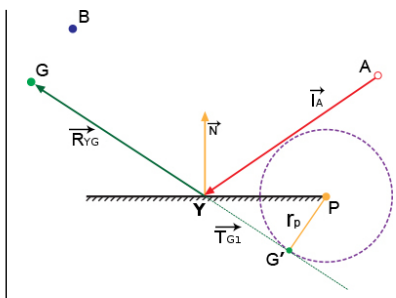

Figure 9

Due to the fact that both $G$ and $B$ belong in the same 2D plane, we can use $B$ to find the two tangents $T_{B_{1}}$ and $T_{B_{2}}$ to the circle defined by the center $P$ and the radius $r_{p}$ (Fig. 10).

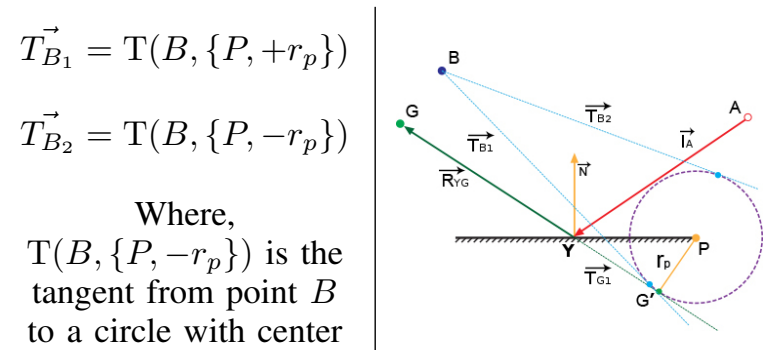

Figure 10
Similar to the tangent $\overrightarrow{T_{G_{1}}}$, each of the two tangents $\overrightarrow{T_{B_{1}}}$ and $\overrightarrow{T_{B_{2}}}$ represent two reversed reflection vectors respectively. By calculating the intersection of $\overrightarrow{I_{A}}$ with $\overrightarrow{T_{B_{1}}}$ and $\overrightarrow{T_{B_{2}}}$, we find two new reflection points $\left(q_{1}, q_{2}\right)$. However, only one of them will produce the correct reflection vector $\overrightarrow{R_{Q B}}$. Using the reflection rule $(\theta i=\theta r)$, we find the point $Q$ by asserting that the angle between $\overrightarrow{I_{A}}$ and the normal $\overrightarrow{N_{q_{1,2}}}$ is equal to the angle formed by $\overrightarrow{T_{B_{1,2}}}$ and $\overrightarrow{N_{q_{1,2}}}$ (Fig. 11).

Reversing the tangent $-\overrightarrow{T_{B_{1}}}$ results into the reflection vector $\overrightarrow{R_{Q B}}$ (Fig. 12). We can now calculate the angle $\phi$ of the mirror by either computing the angle $\angle Y P Q$ or by finding the angle between the two normal vectors $\vec{N}_{q_{1}}$ and $\vec{N}$ (Fig. 12).

The mathematical formula above was extended in order to calculate the rotation of the mirror in a 3-dimensional environment.

$$
\begin{gathered}
\theta i=\angle\left(\overrightarrow{I_{A}} q_{1,2} \overrightarrow{N_{q_{1,2}}}\right), \theta r=\angle\left(\overrightarrow{T_{B_{1,2}}} q_{1,2} \overrightarrow{N_{q_{1,2}}}\right) \\
Q=q_{1,2} \text { iff } \theta i=\theta r
\end{gathered}
$$

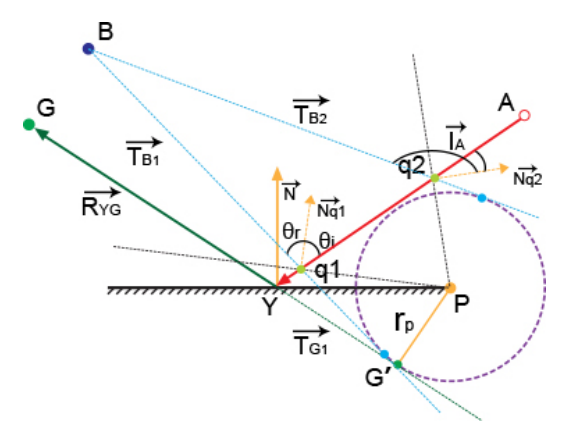

Figure 11

$$
\begin{gathered}
\overrightarrow{R_{Q B}}=-\overrightarrow{T_{B_{1}}} \\
\phi=\angle Y P Q
\end{gathered}
$$

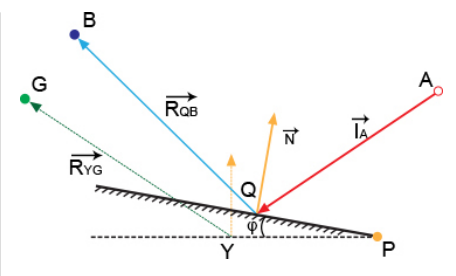

Figure 12

The execution of algorithm begins with the first mirror $M_{1}$ handling the redirection of the light ray from the patient's eyes to the next mirror $M_{2}$. The responsibility of ray retroversion is delegated from one mirror to the other until the last surface.

The intersection of the auxiliary vision vector with the last mirror, returns a reference point to which the third mirror $M_{3}$ needs to retroverse the light ray. The last mirror $M_{4}$ reflects the light ray towards the target locator completing the retroversion. This process occurs for both left and right 
side independently. Figure 13 illustrates the retroversion of the system without considering strabismus, that is, both eyes focus on the same target simultaneously through the device.

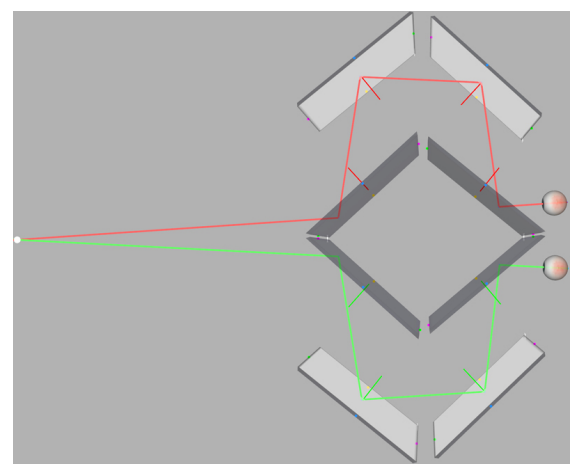

Figure 13: DEMRS retroversion algorithm in use - Top View

The retroversion algorithm does not produce a unique solution as it evaluates the previous mirror angles in order to compute the next. However, due to the initial setup of the mirrors (each at $45^{\circ}$ ), all the returned angles would revolve around that region. The geometric algorithm proposed, is a deterministic solution to the main problem with a $\mathrm{O}(1)$ algorithmic complexity.

\section{SIMULATION RESULTS}

In order to test the DEMRS, we have created a simulated implementation of the described system using a set of strabismus cases for a known target. The location of the fixation point ranges from $0.3 \mathrm{~m}$ to $6.0 \mathrm{~m}$ away in different directions, with the disparity distance assigned to a constant value of $6 \mathrm{~cm}$. At this stage, the system adjusts the mirrors to accomplish horizontal and vertical bifoveal realignment.

Figure 14 illustrates the application of the system for a case where the target (white circle) has been placed straight ahead at $50 \mathrm{~cm}$ away from the center of the eye's disparity (Target $\left._{x, y, z}=\{0.0,0.0,50.0\}\right)$. The "patient" is assumed to suffer from strabismus on the left eye with horizontal and vertical deviation angles of $12^{\circ}$ and $4^{\circ}$, respectively. Since the target is located at less than 6 meters away, the system performs a convergence retroversion on the left side while adjusting the mirrors of the right side to intersect the target.

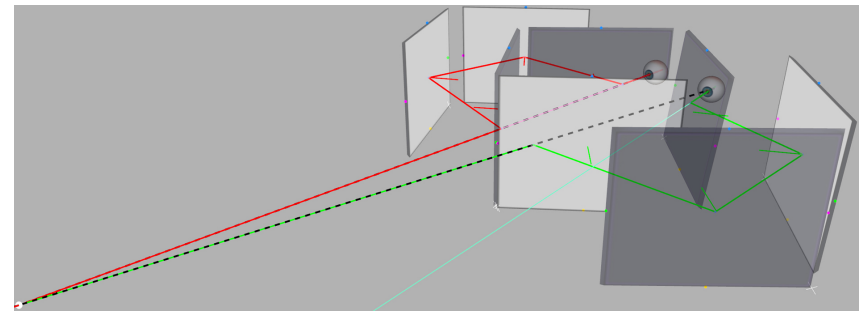

Figure 14: DEMRS result

In several cases of strabismus, such as exotropia, the foveal lines may not intersect at all (Fig. 14). The goal of the
DEMRS is to redirect the vision vectors so that their point of intersection (PoI) is the same as the target's location.

The following tests (Fig. 15, 16) shows the results of the simulated DEMRS with a moving target, changing positions in both horizontal and vertical directions. A number of strabismus angles have been applied on the "bad" eye to evaluate the accuracy of the retroversion algorithm.

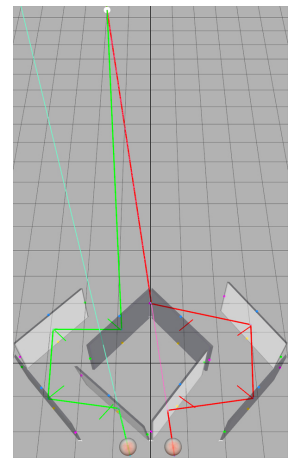

(a) target at left

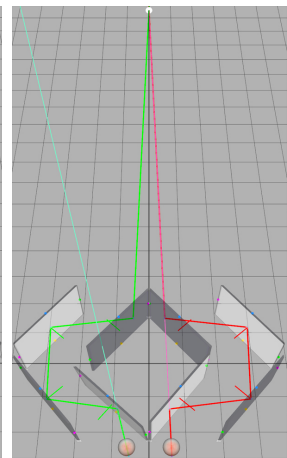

(b) target at center

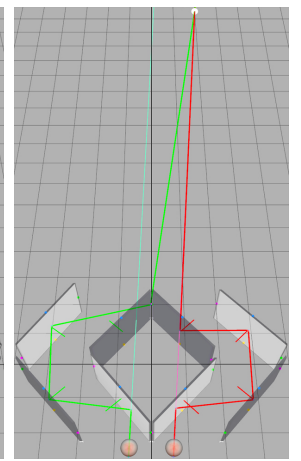

(c) target at right
Figure 15: Top-view results

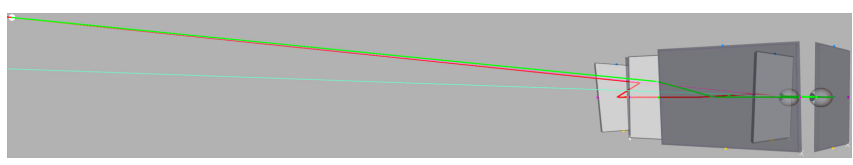

(a) target at top

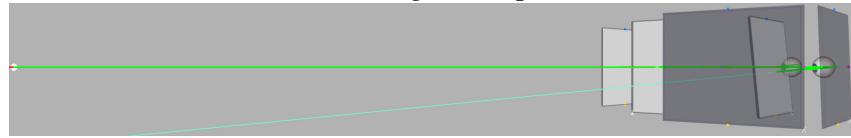

(b) target at center

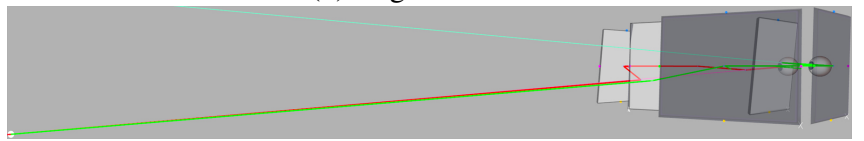

(c) target at bottom

Figure 16: Side-view results

Figure 17 displays the output of the previously explained test (Fig. 14) from a front view, emphasizing on the horizontal and vertical strabismus angles $\left(12^{\circ}, 4^{\circ}\right)$ of the left eye. The redirected vision vectors intersect the target (white circle at the bottom of the figure) successfully.

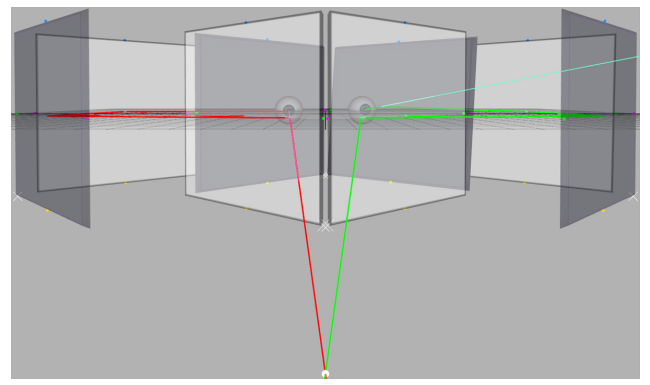

Figure 17: Front-view result 
One of our main concerns during the initial stages of the system's development, was the effect of the device on people without any eye misalignment conditions. Should a system like this have a negative effect on normal vision, then it cannot be considered as a viable diagnosis or treatment tool for strabismus patients either. A basic set up of the device, without the dove prisms, was constructed to test it's applicability on three subjects. Using the mirror handles to adjust their horizontal orientation, each subject was able to perceive the "hidden" image successfully (Fig. 18).

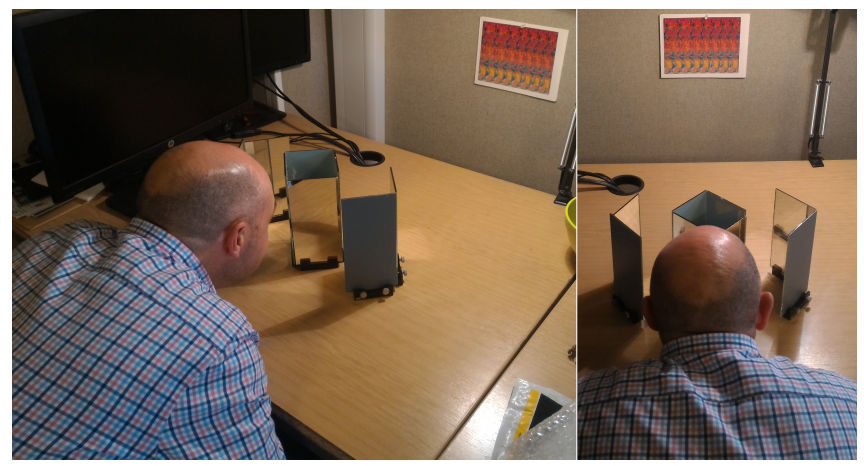

Figure 18: Primitive setup

The DEMRS can successfully re-align the vision vector of a "patient" with strabismus. Both simulated and primitive tests, have returned very promising results and guarantee the correct re-direction of human vision.

\section{FUTURE WORK}

At this stage, the system compensates for a known target located in the range of $0.3 \mathrm{~m}$ to $6.0 \mathrm{~m}$ away. This range forces both eyes to converge on that target. In order to increase the system's flexibility and compensate for a target that is more than $6.0 \mathrm{~m}$ away, the retroversion algorithm needs to adjust the system so that the vision vectors are parallel. Furthermore, the dove or delta prisms would be added to compensate for torsional strabismus.

To extend the system's potential in the areas of diagnosis and treatment, we need to investigate the target deduction method which would allow the system to estimate the target's location.

The DEMRS is a system which needs to work dynamically for different eye angles. This requires for a method to feed the patient's eye angles into the system using an eye tracker. It would allow the DEMRS to continuously re-align the vision vectors in real time.

We can explore the applicability of the device by constructing a basic apparatus that can manually or automatically rotate it's mirrors and prisms.

\section{DISCUSSION}

According to Damanakis (2013) and Hammond (2014), since the DEMRS can support the simultaneous stimulation of the two foveae, it has a great value to patients with constant diplopia [1][3]. Damanakis (2013) confirmed that it can play an important role in the treatment of strabismus as it has the potential to train binocular vision in young children [1]. Both Damanakis (2013) and Hammond (2014) stated that with the current surgery techniques, it is not possible to be exactly accurate when adjusting the eyes [1][3]. The DEMRS would open a huge area towards the actual treatment of strabismus [1][3].

Prof. Hammond (2014) explains that some patients can selectively switch their suppression on and off [3]. In these cases, they can retain their binocularity when looking straight aheaded and suppress it when looking to the side [3]. He then asserts that the DEMRS can maintain their binocularity while medical staff are treating it [3]. Hammond (2014) also believes that "a dynamic act of change in process would be great"[3]. Additionally, compared to an office based diagnosis or treatment method, where motivation is very limited, especially in children [1][3], this system is expected to allow these patients to watch movies or read books at home. Damanakis (2014) concludes that the DEMRS's dynamism, that is it's ability to continuously adjust light rays, is a huge advantage towards the diagnosis of strabismus [2]. Even though vertical disparities are extremely rare, medicine is unable to treat it with surgery [2]. With minor adjustments to the retroversion algorithm, the DEMRS can resolve this issue.

This system was initiated to assist in the diagnosis and treatment of strabismus. However, as Damanakis (2013) and Hammond (2014) have explained, it has the potential to be used in other eye misalignment conditions and other areas of ophthalmology. A prototype of the DEMRS will be developed in order to test it's applicability on patients suffering from strabismus.

\section{ACKNOWLEDGMENT}

We would like to express our sincere gratitude to Prof. Alexandros Damanakis, Prof. Chris Hammond and Dr. Stavros Giannikakis for their most valuable advice and consultation on the medical side of our research.

\section{References}

[1] Glynos C., (2013), "Binocular Vision and Strabismus.", Personal Interview with Dr. A. Damanakis, 31 December 2013, Athens, GR.

[2] Glynos C., (2014), "Methods to estimate the target of a strabismus patient.", Personal Interview with Dr. A. Damanakis, 27 December 2014, Athens, GR

[3] Glynos C., (2014), "The potential use and threats of a Dynamic Eye Misalignment Retroversion System in Strabismus.", Personal Interview with Frost Prof. C. Hammond, 20 March 2014, London, UK.

[4] Glynos, C., (2008), "Eye anatomy and structure", Personal Interview with Dr. S. Giannikakis, 18 December 2008, Athens, GR.

[5] Hubel D., (1995), "Eye, Brain, and Vision”. US: Scientific American Library Series.

[6] Damanakis A., (2004), "Strabismus and oculomotor disturbances", Second Edition, GR: Litsas. 
[7] Julesz B., (1964), "Binocular Depth Perception without Familiarity Cues.", In: SCIENCE, 1964, vol. 145, p.356-362.

[8] Adler P., (2001), "Efficacy of treatment for convergence insufficiency using vision therapy.", In: Opthal. Physiol. Opt., 2002, vol. 22 no. 6, p.565-571.

[9] Barlow B. H., Blakemore C., and Pettigrew D. J., (1967), "The Neural Mechanism of Binocular Depth Discrimination.”, In: Physiol J., 1967, vol. 193, p.327-342.

[10] Cooper J. and Jamal N., (2012), "Convergence Insufficiency a major review.”, In: Optometry, 2012, vol. 83 issue. 4, p.137-158.

[11] Cooper J. and Duckman R., (1977), "Convergence Insufficiency: incidence, diagnosis, and treatment.", In: Journal of the American Optometric Association, 1977, vol. 49 num. 6, p.673-680.

[12] Scheiman M., Mitchell G. L., Cotter S., Cooper J., Kulp M., Rouse M., Borsting E., London R. and Wensveen J., (2005), "A randomized clinical trial of treatments for convergence insufficiency in children.", In: Arch Ophthalmol, 2005, vol. 123 issue. 1, p.14-24.

[13] Cooper J., (2007), "Computerized Vision Therapy for home and office treatment of accommodative \& vergence disorders, \& amblyopia.", In: Journal of Behavioral Optometry, 2007, vol. 18 num. 4, p.88-93.

[14] Carvelho T., Allison R. S., Irving E. L., Herriot, C., (2008), ”Computer gaming for vision therapy", In: IEEE: Virtual Rehabilitation, 2008, p.198-204.

[15] Scott, B. A. (1981), "Botulinum Toxin Injection Of Eye Muscles To Correct Strabismus", In: Trans Am Ophthalmol Soc., 1981, 79, p.734770.

[16] Northing and Easting (2014), "Stereoscopes",

Available from: http://oldtopographer.net/the-topographers-tools/ stereoscopes/ [Accessed: 4 March 2016].

[17] Edmundoptics.co.uk (2016), "Cold Mirrors",

Available from: http://www.edmundoptics.co.uk/optics/opticalmirrors/hot-cold-mirrors/cold-mirrors/1900/ [Accessed: 5 March 2016].

[18] Thorlabs.co.uk (2016), "Mounted Dove Prisms", Available from: http://www.thorlabs.co.uk/newgrouppage9.cfm?object group_id=6810 [Accessed: 5 March 2016]. 